\title{
Evaluation of the effect of diagnostic methodology on the reported incidence of ventilator-associated pneumonia
}

\author{
A Conway Morris, ${ }^{1}$ K Kefala, ${ }^{1}{ }^{12}$ A J Simpson, ${ }^{1}$ T S Wilkinson, ${ }^{1}$ K Everingham, ${ }^{2}$ \\ D Kerslake, ${ }^{2} S$ Raby, ${ }^{2}$ I F Laurenson, ${ }^{3}$ D G Swann, ${ }^{2}$ T S Walsh ${ }^{2}$
}

\section{See Editorial, p 463}

- Additional details of the methodology are published online only at http://thorax.bmi. com/content/vol64/issue6

${ }^{1}$ MRC Centre for Inflammation Research, University of Edinburgh, Scotland, UK

${ }^{2}$ Critical Care, Royal Infirmary of Edinburgh, Scotland, UK:

${ }^{3}$ Department of Clinical

Microbiology, Royal Infirmary of Edinburgh, Scotland, UK

Correspondence to:

Dr A Conway Morris, Room C2.17, MRC Centre for Inflammation Research, Oueen's Medical Research Institute, University of Edinburgh, Little France Crescent, EdinburghEH164TJ,UK; mozza@ doctors.org.uk

Received 27 October 2008 Accepted 26 January 2009 Published Online First

12 February 2009

\section{ABSTRACT}

Background: The optimal method for diagnosing ventilator-associated pneumonia (VAP) is controversial and its effect on reported incidence uncertain. This study aimed to model the impact of using either endotracheal aspirate or bronchoalveolar lavage on the reported incidence of pneumonia and then to test effects suggested from theoretical modelling in clinical practice. Methods: A three-part single-centre study was undertaken. First, diagnostic performance of aspirate and lavage were compared using paired samples from 53 patients with suspected VAP. Secondly, infection surveillance data were used to model the potential effect on pneumonia incidence and antibiotic use of using exclusively aspirate or lavage to investigate suspected pneumonia (643 patients; 110 clinically suspected pneumonia episodes). Thirdly, a practice change initiative was undertaken to increase lavage use; pneumonia incidence and antibiotic use were compared for the 12 months before and after the change.

Results: Aspirate overdiagnosed VAP compared with lavage ( $89 \%$ vs $21 \%$ of clinically suspected cases, $p<0.0001)$. Modelling suggested that changing from exclusive aspirate to lavage diagnosis would decrease reported pneumonia incidence by $76 \%(95 \% \mathrm{Cl} 67 \%$ to $87 \%$ ) and antibiotic use by $30 \%(95 \% \mathrm{Cl} 20 \%$ to $42 \%)$. After the practice change initiative, lavage use increased from $37 \%$ to $58 \%$. Although clinically suspected pneumonia incidence was unchanged, microbiologically confirmed VAP decreased from 18 to 9 cases per 1000 ventilator days $(p=0.001$; relative risk reduction 0.61 (95\% Cl 0.46 to 0.82)), and mean antibiotic use fell from 9.1 to 7.2 antibiotic days (21\% decrease, $p=0.08$ ).

Conclusions: Diagnostic technique impacts significantly on reported VAP incidence and potentially on antibiotic use.

Ventilator-associated pneumonia (VAP) is the most common Intensive Care Unit (ICU)-acquired infection. ${ }^{1}$ Rates of VAP have become markers of "quality of care". ${ }^{23}$

The diagnosis of VAP prompts antibiotic therapy. A diagnostic technique with a high falsepositive rate will increase antibiotic use, potentially resulting in adverse outcomes such as Clostridium difficile-associated diarrhoea and selection pressure for multidrug-resistant bacteria. ${ }^{45}$

The diagnosis of VAP depends on a combination of clinical, radiological and microbiological findings. ${ }^{1}$ Current practice and opinion is divided on the relative merits of non-invasive and invasive (bronchoscopic) techniques in obtaining specimens for diagnosis. A French randomised controlled trial
(RCT) compared an invasive, bronchoscopic diagnostic strategy with a non-invasive strategy using tracheal aspirates. Patients in the invasive diagnostic strategy group had more antibiotic-free days and were more likely to survive to 14 days postrandomisation. $^{6}$ In contrast, a recent Canadian RCT comparing invasive and noninvasive diagnostic strategies found no differences in antibiotic use, or in clinical outcomes. ${ }^{7}$ One possible explanation for these discordant findings could be that diagnostic information was used differently. A positive diagnosis is more likely using endotracheal aspirate (ETA) than bronchoalveolar lavage (BAL), most probably indicating lower specificity. ${ }^{6} 89$ If antibiotics are continued despite negative BAL cultures, any benefits will be lost. Inappropriate early antibiotic therapy is associated with greater mortality,$^{10}{ }^{11}$ which has led to the widespread use of broad-spectrum antibiotics known to cover the major pathogens present in a given ICU. ${ }^{12}{ }^{13}$ The consequences of antibiotic overuse, and specifically failure to de-escalate therapy after negative investigations, are less well understood. Changes in clinical outcomes after altering diagnostic techniques are most likely if linked to clinical decision making, especially in relation to the duration and intensity of antibiotic therapy. ${ }^{14}{ }^{15}$ Using different diagnostic tests could also affect the reported incidence of VAP. Few studies have systematically evaluated this possibility despite the increasing use of VAP incidence as a marker of patient safety and quality of care. ${ }^{23}$

The objective of our study was to use clinical data from our ICU to model the potential effects of diagnostic technique, specifically ETA- versus BALbased diagnosis, on the reported incidence of VAP and associated antibiotic use. We then aimed to evaluate our model by comparing VAP incidence and antibiotic use before and after a practice change initiative designed to increase use of BAL.

\section{METHODS \\ Setting}

The study took place in the 18-bed medicalsurgical critical care unit of a large Scottish teaching hospital. The ICU admits $>1000$ patients annually, of whom $50 \%$ stay for $\geqslant 48 \mathrm{~h}$. The case mix was $50 \%$ surgical in origin, $48 \%$ medical and $2 \%$ obstetric/gynaecological. A total of $83 \%$ of patients received support of two or more organ systems and/or required invasive ventilatory support at some point during their admission. The 
Table 1 Classification of ventilator-acquired pneumonia by the HELICS criteria

\begin{tabular}{ll}
\hline Code & Diagnostic method \\
\hline PN1 & $\begin{array}{l}\text { Positive quantitative culture from minimally contaminated LRT specimen- } \\
\text { BAL } \geqslant 10^{4} \text { CFU }\end{array}$ \\
PN2 & $\begin{array}{l}\text { Positive quantitative culture of LRT (tracheal aspirate) or sputum—not } \\
\text { available within our laboratory }\end{array}$ \\
PN3 & $\begin{array}{l}\text { Positive culture related to no other source—positive pleural fluid culture OR } \\
\text { pulmonary abscess with positive needle aspiration OR positive histology OR } \\
\text { positive exams for virus }\end{array}$ \\
PN4 & $\begin{array}{l}\text { Positive sputum culture or non-quantitative LRT (tracheal aspirate) specimen } \\
\text { culture }\end{array}$ \\
PN5 & No positive microbiology including BAL with $<10^{4}$ CFU
\end{tabular}

All patients meet clinical criteria (radiographic changes, pyrexia or leucocytosis/ leucopenia and clinical signs of chest infection such as increased volume or purulence of sputum, crepitations and deterioration in oxygenation). Adapted from IPSE (Improving Satety in Europe). ${ }^{21}$

BAL, bronchoalveolar lavage; CFU, colony-forming units; HELICS, Hospitals in Europe Link for Infection Control through Surveillance; LRT, lower respiratory tract.

unit is the Scottish Liver Transplant unit (typically 40-50 transplants annually), and receives trauma cases from the region, although isolated neurotrauma was managed elsewhere. During the entire duration of the study, a seven-element ventilator care bundle was in place and remained unaltered. This consisted of routine stress ulcer prophylaxis, head of bed elevation, nurse-led weaning protocol, sedation protocol and scoring, use of heat and moisture exchangers, no routine ventilator circuit changes and early empiric antibiotic treatment for VAP with rationalisation and de-escalation based on the results of cultures. Selective digestive tract decontamination, oral chlorhexadine and subglottic suction were not used at any point during the study.

\section{Overview of study design}

The study had three parts. First, the diagnostic performance of ETA and BAL were compared using paired samples from patients with clinically suspected VAP. Secondly, prospective high quality independently collected infection surveillance data from our ICU were used to model the potential effect on VAP incidence and antibiotic use of exclusively using either ETA or BAL to investigate suspected VAP in our patient population. Thirdly, a practice change initiative was undertaken to increase BAL use. The incidence of VAP and antibiotic use were compared for the 12 months before and after the change using traditional statistical tests, but also statistical process control (SPC) methods.

\section{Comparison of the diagnostic performance of ETA and BAL}

As part of an ongoing study of innate immunity in critically ill patients with suspected VAP, ${ }^{16}$ we collected a series of paired ETA and BAL samples in patients with clinically suspected VAP using a standardised protocol. ${ }^{17} 18$ "True VAP" was defined as culture of organisms in BAL at $>10^{4}$ colony-forming units $(\mathrm{CFU}) / \mathrm{ml} .{ }^{19}$ We compared the diagnostic accuracy of quantitative and non-quantitative cultures of ETA with true VAP. For quantitative ETA a cut-off of $>10^{6} \mathrm{CFU} / \mathrm{ml}^{20}$ was used, whilst any growth was considered positive for qualitative ETA.

\section{Modelling of the potential impact of exclusive use of ETA or BAL on reported VAP incidence and antibiotic use \\ Infection surveillance data}

Since 2005, infection surveillance data on VAP using the Hospitals in Europe Link for Infection Control through Surveillance (HELICS) system ${ }^{21} 22$ has been collected in our
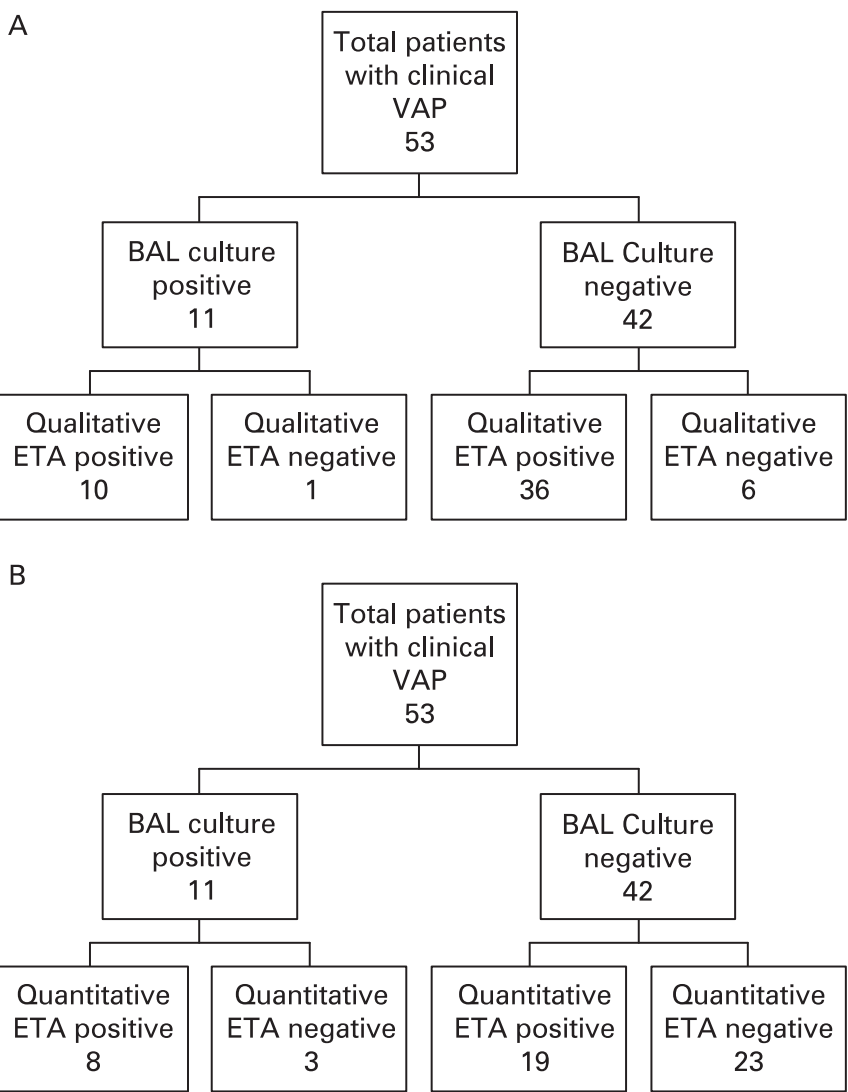

Figure 1 Diagnostic tree for ventilator-associated pneumonia (VAP) by endotracheal aspirate (ETA) using quantitative cultures obtained from rigorously standardised bronchoalveolar lavage (BAL) as the reference. (A) demonstrates this for qualitative cultures, and $(B)$ for quantitative ETA cultures taking $>10^{6}$ colony-forming units (CFU)/ml of ETA fluid as positive.

ICU by an independent infection control team. These included antibiotic prescriptions and diagnostic method (bronchoscopic, pleural or blood culture, qualitative endotracheal cultures, or clinical diagnosis alone). Data for the 12 months prior to the practice improvement intervention (see below) were analysed. The diagnostic categories used by HELICS are shown in table 1

Local intensity of antibiotic use for clinically suspected VAP The "antibiotic load" associated with treating clinically suspected VAP was quantified by calculating the total "antibiotic days" 23 used for each suspected VAP episode. For the purpose of this analysis, we defined this as the number of antibiotics multiplied by the duration of treatment. For instance, if a patient received meropenem for 7 days and vancomycin for 4 days this would equate to 11 "antibiotic days"; concurrent use of two antibiotics counted as two antibiotic days per calendar day. As only qualitative ETA and BAL were in routine clinical use, only these two modalities were compared.

\section{Modelling the theoretical effect of using either ETA or BAL in our patient population}

Using infection surveillance data, the number of clinically suspected VAPs was determined. Using the diagnostic performance characteristics calculated from the paired ETA and BAL samples (see above), we modelled the proportion of clinically suspected VAPs that would have been confirmed 
microbiologically if either BAL, or qualitative ETA or quantitative ETA, had, hypothetically, been used exclusively in our ICU. As a sensitivity analysis we used the 95\% CIs generated from the paired sample data. Using data on antibiotic prescriptions, we also estimated the theoretical effect of changing from exclusively ETA-based diagnosis to BAL-based diagnosis in our ICU.

\section{Prospective evaluation of the effect of increasing the use of BAL on reported VAP incidence and antibiotic use}

We undertook a practice improvement initiative aimed at increasing the use of BAL in our ICU. This included education sessions, improved availability of equipment and expertise for BAL and a case review of VAPs at the weekly grand round. Infection surveillance data were analysed 1 year before and 1 year after the intervention, with a 2-month "run-in period". Specifically we examined the rates of clinically suspected VAP and the rates of microbiologically confirmed VAP, the proportion of patients undergoing bronchoscopic diagnosis and the total number of "antibiotic days" attributable to each episode of VAP. Additional analysis was undertaken using SPC methodology, which allows time series data to be analysed in the context of background variability. ${ }^{24}$ To test whether a change results in a meaningful difference, "warning lines" and "control lines" are established, indicating the upper and lower limits of background variation. If the measured variable crosses these lines it indicates a frequency that is outside normal variation.

\section{Consent and ethical approval}

For the paired ETA and BAL study, witnessed assent was obtained from a relative or main carer for all patients and the study was approved by the Research Ethics Committee. The collection of infection surveillance data did not require ethical approval. Evaluation of the effect of increasing BAL use was considered quality improvement and did not require ethical approval.

Further details of the methodology can be found in the Supplementary data online.

\section{RESULTS}

\section{Diagnostic accuracy of BAL and ETA}

Fifty-three patients had paired samples available for analysis (fig 1). With microbiologically confirmed infection (HELICS criteria PN1; for definitions of HELICS criteria see table 1) the true positive rate for VAP (as defined by quantitative BAL cultures) was $21 \%(\mathrm{n}=11)(95 \%$ CI $10 \%$ to $32 \%)$. Qualitative ETA cultures significantly overdiagnosed VAP compared with BAL; $87 \%(n=46)(95 \%$ CI $77 \%$ to $96 \%)$ of all cultures were positive (HELICS criterion PN4). Quantitative ETA cultures also overdiagnosed VAP, $51 \%(n=27)(95 \%$ CI $37 \%$ to $63 \%)$ of all cultures being positive by this method. A summary of test performance data for ETA compared with BAL is shown in table 2. Although quantitative ETA cultures (using $>10^{6} \mathrm{CFU} / \mathrm{ml}$ as the cut-off) had an effect on sensitivity and specificity, they failed to improve diagnostic performance significantly as assessed by either predictive values or likelihood ratios (table 2). There was no difference in ICU mortality between patients with clinically suspected VAP and positive versus negative BAL cultures $(36 \%$ for both groups). These data suggested that the technique used to diagnose VAP was likely to influence the reported incidence, but did not quantify the likely magnitude of this effect in a clinical population.
Table 2 Diagnostic performance of ETA using quantitative culture obtained from standardised BAL as the reference, with growth at $>$ $10^{4} \mathrm{CFU} / \mathrm{ml}$ defining "true VAP"

\begin{tabular}{lllllll}
\hline & $\begin{array}{l}\text { Sensitivity } \\
(\%)\end{array}$ & $\begin{array}{l}\text { Specificity } \\
(\%)\end{array}$ & $\begin{array}{l}\text { PPV } \\
(\%)\end{array}$ & $\begin{array}{l}\text { NPV } \\
(\%)\end{array}$ & +LR & -LR \\
\hline $\begin{array}{l}\text { Qualitative } \\
\text { ETA cultures }\end{array}$ & 90 & 14 & 21 & 85 & 1 & 0.6 \\
$\begin{array}{l}\text { Quantitative } \\
\text { ETA cultures }\end{array}$ & 72 & 55 & 30 & 88 & 1.6 & 0.5
\end{tabular}

Qualitative ETA is growth of any organism; quantitative ETA is growth of any organism at $>10^{6}$ colony-forming units (CFU) $/ \mathrm{ml}^{20}{ }^{20} \mathrm{~A}$ likelihood ratio is a likelihood that a person with a positive (or negative) test has the disease in question; further detail can be found in Strauss et al. ${ }^{25}$

$\mathrm{BAL}$, bronchoalveolar lavage; ETA, endotracheal aspirate; NPV, negative predictive value; PPV, positive predictive value; $+L R$, positive likelihood ratio; $-L R$, negative likelihood ratio; VAP, ventilator-associated pneumonia.

\section{Modelling of the potential impact of exclusive use of ETA or BAL} on reported VAP incidence and antibiotic use

In the year prior to the intervention (January-December 2006), 643 patients were admitted to the ICU for $>48 \mathrm{~h}$, corresponding to 3771 ventilator days. There were 110 episodes of clinically suspected VAP, based on HELICS criteria. Of these, 68 had positive microbiological cultures. The classification of these VAPs based on the HELICS system is shown in fig 2. The incidence of clinically suspected VAP was 17\% (29.1 cases per 1000 ventilator days) and microbiologically confirmed VAP $10.5 \%$ (18 cases per 1000 ventilator days). The associated antibiotic use with each mode of diagnosis is shown in table 3 . A total of $16 \%$ of patients had their antibiotics changed in ICU in the $72 \mathrm{~h}$ prior to diagnosis, an acknowledged risk factor for false-negative cultures. ${ }^{1} \mathrm{~A}$ total of $89 \%$ of patients had received antibiotics prior to diagnosis, $75 \%$ within $72 \mathrm{~h}$ of diagnosis.

\section{Modelled effect on reported incidence}

Based on the above data and test-specific data from the paired samples study (with $95 \%$ CI as sensitivity analysis from fig 1), the potential impact of using exclusively ETA or BAL for

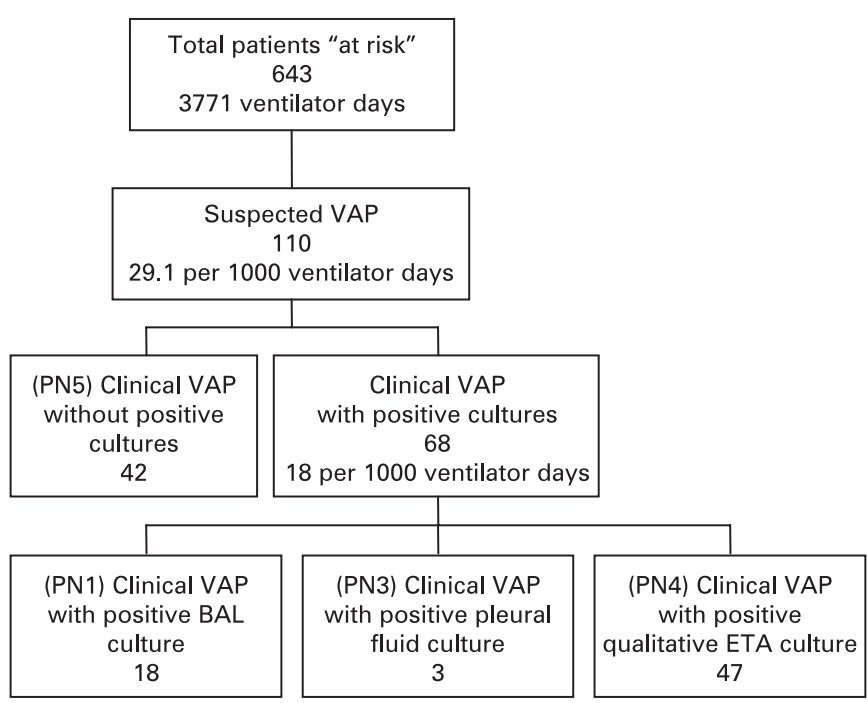

Figure 2 Data from routine infection surveillance. Patients at risk of ventilator-associated pneumonia (VAP) are all those mechanically ventilated for $\geqslant 48 \mathrm{~h}$. PN numbers refer to the criteria laid out in the HELICS (Hospitals in Europe Link for Infection Control through Surveillance) protocol (see table 1). BAL, bronchoalveolar lavage; ETA, endotracheal aspirate. 
Table 3 Treatment intensity as measured by "antibiotic days" (ie, number of antibiotics prescribed multiplied by the duration of treatment)

\begin{tabular}{llll}
\hline & $\begin{array}{l}\text { Positive sample } \\
\text { result }\end{array}$ & $\begin{array}{l}\text { Negative sample } \\
\text { result* }\end{array}$ & $\begin{array}{l}\text { Overall antibiotic } \\
\text { use associated } \\
\text { with diagnostic } \\
\text { technique }\end{array}$ \\
\hline $\begin{array}{l}\text { Qualitative ETA } \\
\text { diagnosis }\end{array}$ & $11.5(8.9$ to 14$)$ & $1.5(0$ to 5$)$ & $9.9(7.4$ to 12.3$)$ \\
BAL diagnosis & $12.1(8.8$ to 15$)$ & $5.8(3.7$ to 7.9$)$ & $7.9(6$ to 9.8$)$ \\
\hline
\end{tabular}

Data shown as mean and $95 \% \mathrm{Cl}$.

${ }^{*}$ Sterile for ETA, $<10^{4}$ colony-forming units $(\mathrm{CFU}) / \mathrm{ml}$ for BAL.

$\mathrm{BAL}$, bronchoalveolar lavage; ETA, endotracheal aspirate.

diagnosis on the reported incidence of microbiologically confirmed VAP in our ICU is shown in table 4. These data suggested that if BAL were used exclusively together with HELICS surveillance definitions of VAP (PN1), the reported incidence of VAP in our population would be $76 \%$ (95\% CI $67 \%$ to $87 \%$ ) lower than if non-quantitative ETA were used exclusively (PN4) (relative risk reduction 0.22 (95\% CI 0.17 to $0.35)$ ). This estimate did not take into account the small (1\%) false-negative rate with qualitative ETA cultures. Use of BAL in preference to a quantitative ETA culture (PN2) would result in a $59 \%$ ( $95 \%$ CI $50 \%$ to $73 \%$ ) reduction, but quantitative ETA cultures would have a $6 \%$ false-negative rate (and, as can be seen in table 2, quantitative ETA culture did not have significantly better diagnostic performance than qualitative ETA culture).

\section{Modelled effects on antibiotic use}

Using the actual rates of antibiotic use associated with different diagnostic techniques (table 3 ) and the test performance documented in the paired sample analysis (table 4), the hypothetical effects of exclusively using either ETA or BAL on total antibiotic use are shown in table 5 . This model suggested that antibiotic use could be reduced by up to $30 \%$ (95\% CI $20 \%$ to $42 \%$ ) in our ICU by using BAL rather than ETA if clinical use of antibiotics in response to positive and negative ETA and BAL data did not change.

\section{Prospective evaluation of the effect of increasing the use of BAL on reported VAP incidence and antibiotic use}

The patient case mix was similar in both the preintervention and postintervention periods (table 6). Following the intervention, the rate of bronchoscopy in suspected VAP increased from

Table 4 Modelled effect of exclusive use of ETA or BAL on the reported incidence of VAP, using the numbers of clinically suspected VAP episodes from infection surveillance occurring in the ICU over 12 months (fig $2 ; n=110$ )

\begin{tabular}{llll}
\hline & $\begin{array}{l}\text { BAL mean } \\
\text { estimate } \\
\text { (95\% CI) }\end{array}$ & $\begin{array}{l}\text { Qualitative ETA } \\
\text { mean estimate } \\
\text { (95\% CI) }\end{array}$ & $\begin{array}{l}\text { Quantitative ETA } \\
\text { mean estimate } \\
\text { (95\% CI) }\end{array}$ \\
\hline $\begin{array}{l}\text { Positive culture } \\
\text { Negative culture }\end{array}$ & $\begin{array}{l}87(11 \text { to } 35) \\
(75 \text { to } 99)\end{array}$ & $\begin{array}{l}96(85 \text { to } 106) \\
14 \text { to } 25)\end{array}$ & $\begin{array}{l}56(41 \text { to } 69) \\
54(41 \text { to } 69)\end{array}$ \\
$\begin{array}{l}\text { Proportion of } \\
\text { "clinically } \\
\text { suspected VAPs" } \\
\text { reported as } \\
\text { "confirmed VAP" }\end{array}$ & $21 \%(10$ to $32 \%)$ & $87 \%(77$ to $96 \%)$ & $51 \%(37$ to $63 \%)$ \\
$\begin{array}{l}\text { Cases/1000 } \\
\text { ventilator days }\end{array}$ & $6.1(2.9$ to 9.2) & $25.5(22.5$ to 28.1) & $15.0(11.0$ to 18.3) \\
\hline
\end{tabular}

Numbers show mean estimate and upper and lower confidence limit using 95\% Cls for proportions ( $\mathrm{z}$ test) and were derived using the data from fig 1.

$\mathrm{BAL}$, bronchoalveolar lavage; ETA, endotracheal aspirate; ICU, Intensive Care Unit; $\mathrm{VAP}$, ventilator-associated pneumonia.
$37 \%$ to $58 \%$ (68\% in the final quarter of the analysis period). The overall rate of clinically suspected VAP was similar before and after the intervention (table 6). In contrast, there was a clinically and statistically significant decrease in the reported incidence of confirmed VAP during the 12 months following the practice change, (relative risk reduction 0.61 (95\% CI 0.46 to 0.82) $p=0.0012$; table 6). Antibiotic use decreased by $21 \%$, which was clinically important (1.9 fewer antibiotic days per episode during the second 12 -month period), but did not reach statistical significance (table 6; $p=0.08$ ). There was no difference in the proportion of patients who had antibiotics changed in the $72 \mathrm{~h}$ before diagnosis (16\%), nor in the proportion receiving antibiotics prior to diagnosis (89\%, 70\% within $72 \mathrm{~h}$ of diagnosis). Mortality for all patients with clinically suspected VAP fell from $37.5 \%$ to $17 \%(p=0.002)$, although the median APACHE II (Acute Physiology and Chronic Health Evaluation II) score in the patients with clinically suspected VAP was lower in the postintervention period (23 vs $20 \mathrm{p}=0.02$ ). These data indicated changes consistent with those predicted in our hypothetical model, particularly as the difference in the use of ETA versus BAL was $21 \%$ compared with $100 \%$ in the model.

The reported incidence of VAP using HELICS surveillance methodology over the entire period of evaluation (January 2006 to May 2008) are shown using SPC charting in fig 3. Of note, there were two time points when the incidence of VAP crossed the upper warning line, indicating variation above normal background variability, during the preintervention period and no episodes following it. Towards the latter part of the postintervention period the incidence dropped below the longterm average for our unit, suggesting a genuine reduction in reported incidence of VAP (for an in-depth review of SPC methodology, see Beneyan et al. ${ }^{24}$

\section{DISCUSSION}

This study provides the first evaluation of the effect of changing the method of diagnosis on the rate of VAP within the context of normal clinical practice. Using a combination of ongoing surveillance data and direct comparisons of ETA and BAL performance in our own population, we modelled the likely effect of increasing the use of BAL on VAP rates and antibiotic use. We then confirmed this effect using a practice change initiative. Our data suggest a favourable effect on both reported VAP rates and antibiotic use from increasing BAL use in routine clinical practice. The changes were consistent with the effects predicted by the model given that the clinical change in diagnostic method was significantly smaller than the hypothetical model (21\% vs $100 \%$ ).

A strength of our study was the use of high quality infection surveillance data of routine clinical care collected by an independent infection control team, so reducing the risk of bias. The modelling of the potential impact of practice change was conducted using high quality samples from a single experienced bronchoscopist using a highly standardised procedure. All data used in the modelling process were derived from the unit in which the practice improvement occurred, which minimised the number of assumptions that had to be made. By conducting a sensitivity analysis we were able to explore the potential range of effects.

The modelling was tested in the same institution through the use of a quality improvement approach, thus embedding the evaluation within routine practice. Analysis of the results was conducted by classic statistical tests and SPC methodology which both demonstrated similar effects. 
Table 5 Modelled effect of changing diagnostic strategy on the antibiotic load experienced by patients with suspected VAP

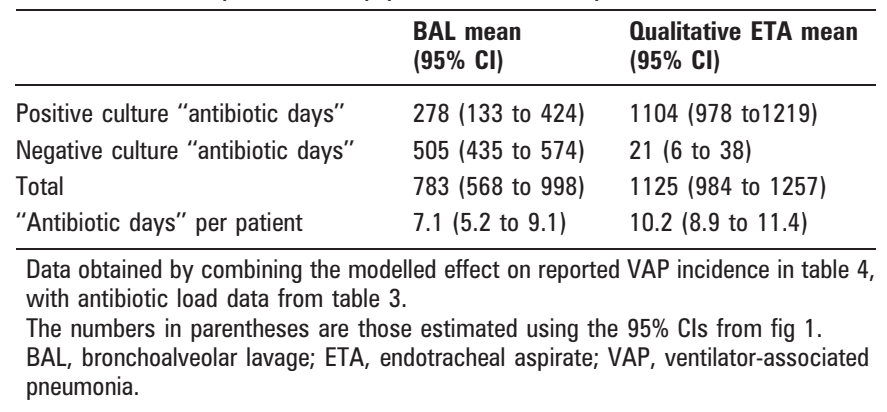

Debate continues around the mortality that can be attributed to $\mathrm{VAP}^{14}$ and the value of using this as an outcome measure in studies, ${ }^{15}$ with a suggestion that other measures such as antibiotic use should be used. The reduction in antibiotics in our study did not achieve statistical significance, although there was a strong trend towards this. The study was not powered to detect a difference in antibiotic prescription, but rather was a pragmatic investigation in the context of normal clinical practice.

Caution should be exercised in extrapolating these data to other populations. The use of a before and after study design is subject to potential time and treatment effect biases, although the finding of similar results to those predicted by the modelling is encouraging. We have compared ETA with a test that is not a universally accepted gold standard (ie, quantitative BAL). In comparison with the proposed, but clinically impractical, gold standard of histology, quantitative BAL may have only moderate predictive ability. ${ }^{26}{ }^{27}$ However, our comparison was pragmatic and clinically relevant.

The lack of specificity of ETA cultures has been noted previously $^{8}$ and this is reflected in the higher proportion of diagnoses in patients whose samples are ETA derived. ${ }^{6}$ Some studies have suggested no difference in sensitivity between the two techniques, ${ }^{28}{ }^{29}$ and consequently no impact on treatment or outcomes. ${ }^{7}$ Although some studies have suggested that quantitative cultures of ETA can be used in place of invasive techniques, ${ }^{20} 30{ }^{31}$ we did not demonstrate sufficient diagnostic performance to support this approach, and the $6 \%$ rate of false negatives gives cause for concern.

Clinical criteria alone are problematic in the diagnosis of VAP, as a variety of pathologies can mimic pneumonia. ${ }^{32}$ Attempts to develop more structured clinical tools such as the Clinical Pulmonary Infection Score (CPIS) have not demonstrated significantly improved diagnosis, ${ }^{33}$ although they may have a role in determining response to therapy. ${ }^{14}$ Our study confirms the low specificity of clinical criteria.

The finding of reduced reported incidence with BAL-based strategies has two possible interpretations, which are relevant to the conflicting conclusions of the two major RCTs in the field. ${ }^{6}$ First, it may be that quantitative BAL is underdiagnosing true VAP, and that the apparent reduction in incidence and reduction in antibiotic use represent missed VAPs and hence undertreatment. The second interpretation is that ETA overdiagnoses VAP and that use of this method results in overtreatment. Were the former to be correct one might expect a reduction in antibiotic use to be associated with an increased mortality or duration of ventilation. In our study we found a reduction in mortality. There are several possible explanations

Table 6 Demographic characteristics of the populations of ICU patients admitted during the preintervention and postintervention periods, of the patients with clinically suspected VAP and the effects of the intervention on diagnosis of VAP and antibiotic use

\begin{tabular}{|c|c|c|c|c|}
\hline & $\begin{array}{l}\text { Preintervention } \\
\text { period }\end{array}$ & $\begin{array}{l}\text { Postintervention } \\
\text { period }\end{array}$ & Difference (95\% CI) & p Value \\
\hline \multicolumn{5}{|l|}{ Entire population admitted to ICU } \\
\hline Total number of patients & 1059 & 1075 & & \\
\hline$\%$ male & $58 \%$ & $59 \%$ & $-1 \%(-5 \%$ to $3 \%)$ & $0.43^{*}$ \\
\hline Mean age (years) & 55.9 & 54.4 & $1.5(0$ to 3$)$ & $0.06 \dagger$ \\
\hline Median (IQR) APACHE II score & $17(12-23)$ & $17(11-23)$ & 0 & $0.91 \%$ \\
\hline Median (IQR) length of stay (days) & $1.9(0.9-4.9)$ & $2.1(0.9-4.85$ & 0.2 & $0.78 \%$ \\
\hline ICU mortality & $19 \%$ & $20 \%$ & $-0.7 \%(-4 \%$ to $2.6 \%)$ & $0.6^{*}$ \\
\hline $\begin{array}{l}\text { Patients "at risk" (ie, length of stay } \\
\geqslant 48 \mathrm{~h} \text { ) }\end{array}$ & 643 & 667 & & \\
\hline Number of at-risk "ventilator days" & 3771 & 3777 & & \\
\hline \multicolumn{5}{|c|}{ Population with clinically suspected VAP } \\
\hline Number with clinically suspected VAP & $110(17 \%)$ & $94(14 \%)$ & $-3 \%(-6 \%$ to $1 \%)$ & $0.16^{*}$ \\
\hline $\begin{array}{l}\text { Incidence of suspected VAP (per } 1000 \\
\text { ventilator days) }\end{array}$ & 29.1 & 24.9 & & \\
\hline Median (IQR) APACHE II score & $23(18-26.5)$ & $20(16-24)$ & 3 & $0.02 \%$ \\
\hline $\begin{array}{l}\text { Number of suspected VAPs undergoing } \\
\text { bronchoscopic diagnosis }\end{array}$ & $41(37 \%)$ & $53(58 \%)$ & $19 \%(5$ to $32 \%)$ & $0.004^{*}$ \\
\hline $\begin{array}{l}\text { Cases of microbiologically confirmed } \\
\text { VAP }\end{array}$ & $68(62 \%)$ & $36(40 \%)$ & $-22 \%(-36 \%$ to $-10 \%)$ & $0.0012^{*}$ \\
\hline$\%$ Gram-negative organisms & $51 \%$ & $62 \%$ & $11 \%(-37 \%$ to $15 \%)$ & $0.35^{*}$ \\
\hline $\begin{array}{l}\text { Incidence of confirmed VAP (per } 1000 \\
\text { ventilator days) }\end{array}$ & 18 & 9 & & \\
\hline Mean "antibiotic days" & 9.1 & 7.2 & $-1.8(-3.8$ to 0.4$)$ & $0.08 \dagger$ \\
\hline Mortality & $37.5 \%$ & $17 \%$ & $-20.5 \%(-22 \%$ to $-19 \%)$ & $0.003^{*}$ \\
\hline 1Median (IQR) duration of ventilation & $16(10-30)$ & $19(10-27)$ & 3 & 0.6 \\
\hline
\end{tabular}

*By z test for proportions. †By t test. \$By Mann-Whitney U test.

APACHE II, Acute Physiology and Chronic Health Evaluation II; BAL, bronchoalveolar lavage; ETA, endotracheal aspirate; ICU, Intensive Care Unit; IQR, interquartile range; VAP, ventilator-associated pneumonia. 
Figure 3 Statistical process chart showing incidence of confirmed ventilator-associted pneumonia (VAP) in the preintervention and postintervention periods. Process av, process average; UCL, upper control line; UWL, upper warning line. Lower control line and lower warning lines are omitted for clarity.

$$
\rightarrow-V A P \quad-\text { UCL } \cdots \cdot \text { UWL }- \text { Process av }
$$

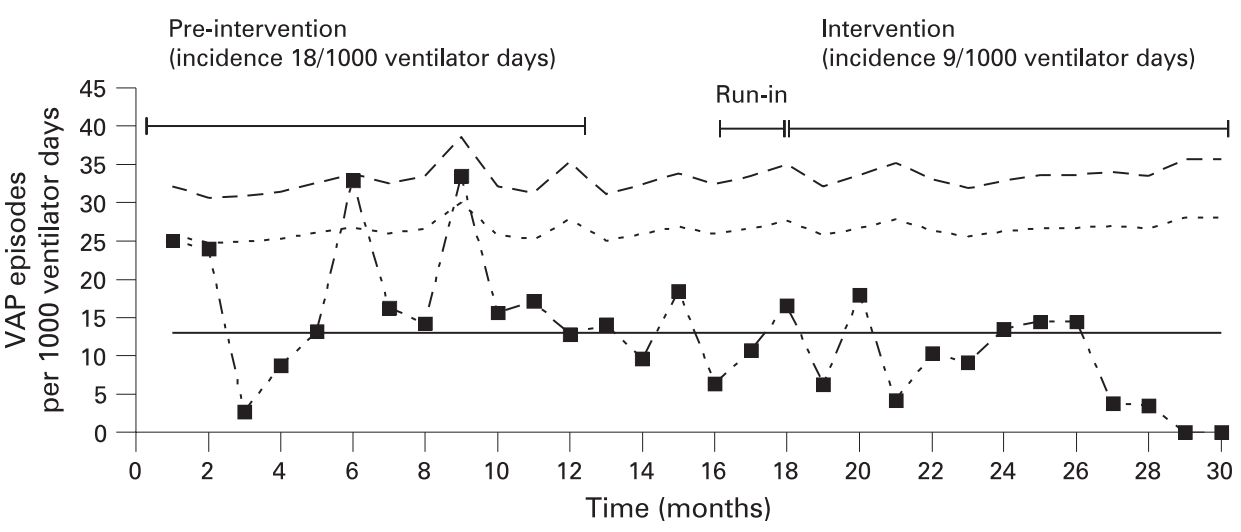

Pre-intervention

(incidence $18 / 1000$ ventilator days) for this observation. It is possible that the case mix was different, despite comparing all patients fulfilling clinical HELICS VAP criteria (a similar proportion in both cohorts). Illness severity at ICU admission was higher in the preintervention group, as indicated by higher APACHE II scores, which is likely to account for some of the observed effect. However, the observed reduction in mortality associated with clinically suspected VAP is consistent with a previous RCT $^{6}$ and other studies $^{34}$ showing associations between inappropriate antibiotic therapy and increased mortality. It is not possible from our before and after study to be sure of the relative contribution, if any, to reduced mortality from changing diagnostic methodology.

Much of the debate concerning methods of diagnosis of VAP has revolved around the impact on patient outcomes. ${ }^{15}$ This is the first study to look specifically at the effect on incidence rates, which are of crucial importance to intraunit and interunit comparisons. These form the cornerstones of "benchmarking" in the quality improvement processes. ${ }^{2}{ }^{3}$ With the potential for the withdrawal of reimbursement by Medicaid and Medicare for episodes of VAP in US hospitals, variations in incidence rates may also have significant financial impact. The variability in incidence demonstrated above leads us to question the usefulness of comparisons of VAP rates for quality control, unless there is considerably more standardisation of diagnostic techniques.

We have demonstrated that, in a unit that is receptive to the idea, the bronchoscopy rate in suspected VAP can be increased and that this increase leads to a significant decrease in reported incidence. Alongside this is a reduction in antibiotic use, suggesting that it is what the clinician does with the information derived from diagnostic testing that is of crucial importance. Despite most infections being monobacterial, especially amongst those who underwent diagnosis by BAL, most patients initially received a combination of antibiotics, with de-escalation once the infective organism was confirmed by microbiological culture. This is in accordance with suggested antimicrobial best practice. ${ }^{12} 13$ In the future the use of more rapid diagnostic strategies such as PCR testing ${ }^{35}$ may allow deescalation to occur more promptly, so further reducing antibiotic exposure. The finding that our unit tended to use shorter courses of antibiotics for negative ETA samples than for negative BAL samples is intriguing. This study was not designed to look at clinicians' decision-making processes. It is possible that there were differences between patients selected for bronchoscopy and those who were not. Alternatively, clinicians may have greater confidence that a negative ETA, which in our unit meant no bacterial growth, reflected a true negative for pneumonia. A negative BAL, where bacterial growth below the threshold of $10^{4} \mathrm{CFU} / \mathrm{ml}$ was classed as negative, may have been associated with greater clinical uncertainty.

A number of interventions have been proposed to reduce rates of VAP, which have been included in "ventilator care bundles", promoted by a growing number of quality improvement organisations. ${ }^{2}{ }^{3}$ The results of this study suggest that increasing the use of bronchoscopy as the preferred diagnostic modality may significantly reduce reported VAP rates. This is clearly relevant to any research in this area, and future trials into diagnostic technique should take into account how the information from these tests is applied by clinicians. It is also vital that comparisons between units take account of differences in diagnostic technique used, and that changes in diagnostic technique be reported alongside any apparent changes in incidence.

Acknowledgements: Thanks are due to the staff of the Wellcome Trust Clinical Research Facility, Edinburgh, for their creation of the data capture tool used in the antibiotic use survey. Thanks are also due to the staff of the Intensive Care Unit and Clinical Microbiology Department at the Royal Infirmary of Edinburgh.

Funding: This work was supported by a grant from the Sir Jules Thorn Charitable Trust, an unrestricted educational grant from Wyeth Pharmaceuticals and the Edinburgh Critical Care Research Group Trials fund.

Competing interests: AJS has received expenses from Astra Zeneca and Glaxo Smith Kline (for travel and accommodation) to attend international educational conferences. IFL has received expenses from Astra Zeneca (for travel and accommodation) to attend international educational conferences. TSW is the recipient of an unrestricted educational grant from Wyeth pharmaceuticals for work concerning epidemiology of ICU-acquired infection. All other authors have no conflicts of interest to declare.

Patient consent: The study was approved by the Research Ethics Committee.

\section{REFERENCES}

1. Chastre J, Fagon J-Y. Ventilator-associated pneumonia. Am J Respir Crit Care Med 2002;165:867-903.

2. www.ihi.org/lHI/Topics/CriticalCare/IntensiveCare/Changes/Implementthe2. VentilatorBundle.htm (accessed 30 September 2008).

3. www.patientsafetyalliance.scot.nhs.uk/ (accessed 30 September 2008).

4. Wiström J, Norrby SR, Myhre EB, et al. Frequency of antibiotic-associated diarrhoea in 2462 antibiotic-treated hospitalized patients: a prospective study. J Antimicrob Chemother 2001;47:43-50.

5. Dancer SJ, Coyne M, Robertson C, et al. Antibiotic use is associated with resistance of environmental organisms in a teaching hospital. J Hosp Infect 2006;62:200-6.

6. Fagon J-Y, Chastre J, Wolff $\mathrm{M}$, et al. Invasive and non-invasive strategies for management of suspected ventilator-associated pneumonia. A randomized trial. Ann Intern Med 2000:132:621-30. 
7. Canadian Critical Care Trials Group. A randomized trial of diagnostic techniques for ventilator-associated pneumonia. N Engl J Med 2006;355:2619-30.

8. Cook D, Mandell L. Endotracheal aspiration in the diagnosis of ventilator-associated pneumonia. Chest 2000:117:195S-7S.

9. Lambert RS, Vereen LE, George RB. Comparison of tracheal aspirates and protected brush catheter specimens for identifying pathogenic bacteria in mechanically ventilated patients. Am J Med Sci 1989;297:377-82.

10. Iregui M, Ward S, Sherman G, et al. Clinical importance of delays in the initiation of appropriate antibiotic treatment for ventilator-associated pneumonia. Chest 2002;122:262-8.

11. Kollef KE, Schramm GE, Wills AR, et al. Predictors of 30-day mortality and hospital costs in patients with ventilator-associated pneumonia attributed to potentially antibiotic-resistant Gram-negative bacteria. Chest 2008;134:281-7.

12. American Thoracic Association and Infectious Diseases Society of America. Guidelines for the management of adults with hospital-acquired, ventilatorassociated, and healthcare-associated pneumonia. Am J Respir Crit Care Med 2005; 171:388-416.

13. Masterton RG, Galloway A, French G, et al. Guidelines for the management of hospital-acquired pneumonia in the UK: report of the working party on hospitalacquired pneumonia of the British Society for Antimicrobial Chemotherapy. J Antimicrob Chemother 2008:62:5-34

14. Kollef MH, Morrow LE, Niederman MS, et al. Clinical characteristics and treatment patterns among patients with ventilator-associated pneumonia. Chest 2006;129:1210-8.

15. Wunderink RG. Mortality and the diagnosis of ventilator-associated pneumonia: a new direction. Am J Respir Crit Care Med 1998:157:349-50.

16. Conway Morris A, Kefala K, Wilkinson TS, et al. C5a mediates peripheral blood neutrophil dysfunction in critically ill patients. Am J Respir Crit Care Med 2009, Mar 26. [Epub ahead of print]

17. Meduri GU, Chastre J. The standardization of bronchoscopic techniques for ventilator-associated pneumonia. Chest 1992;102:557s-63s.

18. Baselski VS, Wunderink RG. Bronchoscopic diagnosis of pneumonia. Clin Microbiol Rev 1994;7:533-58.

19. Chastre J, Fagon JY, Bornet-Lecso M, et al. Evaluation of bronchoscopic techniques for the diagnosis of nosocomial pneumonia. Am J Respir Crit Care Med 1995; 152:231-40.

20. Marquette $\mathbf{C H}$, Georges $\mathrm{H}$, Wallet F, et al. Diagnostic efficiency of endotracheal aspirates with quantitative bacterial cultures in intubated patients with suspected pneumonia. Comparison with the protected specimen brush. Am Rev Respir Dis 1993:148:138-44.

21. http://helics.univ-lyon1.fr/ (accessed 30 September 2008).

22. Suetens C, Morales I, Savey A, et al. European surveillance of ICU-acquired infections (HELICS-ICU): methods and main results. J Hosp Infect 2007;65:171-3.

23. Swoboda SM, Dixon T, Lipsett PA. Can the clinical pulmonary infection score impact ICU antibiotic days? Surg infect 2006:7:331-9.

24. Benneyan JC, Lloyd RC, Plsek PE. Statistical process control as a tool for research and healthcare improvement. Qual Saf Health Care 2003;12:458-64.

25. Strauss S, Richardson WS, Glasziou P, et al. Evidence based medicine. 3rd edn. Oxford: Churchill Livingstone , 2005

26. Marquette $\mathbf{C H}$, Copin $\mathrm{MC}$, Wallet $\mathrm{F}$, et al. Diagnostic tests for pneumonia in ventilated patients: prospective evaluation of diagnostic accuracy using histology as a diagnostic gold standard. Am J Respir Crit Care Med 1995;151:1878-88

27. Rouby JJ, De Lassale EM, Poete P, et al. Nosocomial bronchopneumonia in the critically ill. Histologic and bacteriologic aspects. Am Rev Respir Dis 1992;146:1059-66.

28. Solé Violán J, Fernández $\mathrm{JA}$, Benítez $\mathrm{AB}$, et al. Impact of quantitative invasive diagnostic techniques in the management and outcome of mechanically ventilated patients with suspected pneumonia. Crit Care Med 2000;28:2737-41.

29. Ruiz M, Torres A, Ewig S, et al. Non-invasive versus invasive microbial investigation in ventilator-associated pneumonia: evaluation of outcome. Am J Respir Crit Care Med 2000;162:119-25.

30. Liang Wu C, Yang DI, Wang NY, et al. Quantitative culture of endotracheal aspirates in the diagnosis of ventilator-associated pneumonia in patients with treatment failure Chest 2002;122:662-8.

31. Torres A, Martos A, Puig de la Bellacasa J, et al. Specificity of endotracheal aspiration, protected specimen brush, and bronchoalveolar lavage in mechanically ventilated patients. Am Rev Respir Dis 1993;147:952-7.

32. Meduri GU, Mauldin GL, Wunderink RG, et al. Causes of fever and pulmonary densities in patients with clinical manifestations of ventilator-associated pneumonia. Chest 1994;106:221-35.

33. Schurink M, Van Nieuwenhoven CA, Jacobs JA, et al. Clinical pulmonary infection score for ventilator-associated pneumonia: accuracy and inter-observer variability. Intensive Care Med 2004;30:217-24.

34. Yu VL, Singh N. Excessive antimicrobial usage causes measurable harm to patients with suspected ventilator-associated pneumonia. Intensive Care Med 2004;30:735-8.

35. Strålin K, Korsgaard J, Olcén P. Evaluation of a multiplex PCR for bacterial pathogens applied to bronchoalveolar lavage. Eur Respir J 2006;28:568-75.

\section{Lung alert}

\section{Genetic determinants of lung cancer micrometastasis}

Complete resection of non-small cell lung cancer without lymph node or distant metastasis has a relapse rate within 2 years of surgery of approximately $40 \%$. This study is the first to show a pattern of genomic profiles that appear to be specific to early micrometastasis.

Primary tumour cells were collected from 62 patients with non-small cell lung cancer undergoing surgical resection. These were subsequently divided into two groups based on the absence or presence of disseminated tumour cells (DTC) in bone marrow using immunocytochemical staining. Genomic aberrations were detected by microarray and fluorescence in situ hybridisation (FISH) analyses.

The most significant finding was loss of a region of chromosome $4 \mathrm{q}$ in bone marrow-positive patients and a gain in bone marrow-negative cases. Additional FISH analysis was also performed on a tissue microarray of 36 brain metastases of the lung, with $39 \%$ of samples showing a one allele loss of $4 \mathrm{q}$. $4 \mathrm{q}$ loss was more commonly found in squamous cell carcinoma than adenocarcinomas. Brain metastases showed more losses of 4q21 than primary lung tumours overall.

The authors conclude that lung cancer development is a multistep genomic process and a $4 \mathrm{q}$ loss has a highly significant association with bone marrow-positive status. Further studies are required in this highly complex area before genomic aberrations can be used as a prognostic marker in the treatment of lung cancer.

- Wrage M, Ruosaari S, Eijk P, et al. Genomic profiles associated with early micrometastasis in lung cancer: relevance of 4q deletion. Clin Cancer Res 2009;15:1566-74.

\section{S Rowan}

Correspondence to: Dr S Rowan, StR4 Respiratory Medicine, Altnagelvin Hospital, Londonderry, Northern Ireland; stephen_rowan@hotmail.com 